\title{
Electroweak fits at LEP
}

\author{
E. Lançon ${ }^{\mathrm{a} *}$ \\ ${ }^{\text {a} D A P N I A / S P P, ~ C E A-S a c l a y, ~}$ \\ 91191 Gif-sur-Yvette Cedex, France
}

High precision electroweak measurements performed over ten years at LEP and SLC have allowed to constrain the Standard Model of electroweak interactions. The model have been used to predict the mass of the top quark and to set limits on the mass of the Higgs boson.

\section{Why electroweak fits?}

Electroweak fits have been extensively used at LEP and SLC for testing the Standard Model at the level of its quantum corrections, searching for deviations that may signal the presence of new physics. They have also successively predicted the top quak mass prior to its discovery and recently, allowed to set limit on the Higgs boson mass. Sensitivity to top quark and Higgs boson masses can be infered from the following exemple relation :

$m_{\mathrm{W}}^{2}\left(1-\frac{m_{\mathrm{W}}^{2}}{m_{\mathrm{Z}}^{2}}\right)=\frac{\pi \alpha}{\sqrt{2} G_{\mathrm{F}}}(1+\Delta r)$

where $\Delta r$ contains several terms: $\Delta \alpha$ (due to light fermion masses), a negative term (several $\%$ ) proportional to $m_{\mathrm{t}}^{2}$ and a relatively small (below 1\%) term proportional to $\ln \left(m_{\mathrm{H}}\right)$. Therefore, the inferred constraints on $m_{\mathrm{H}}$ are much weaker than those on $m_{\mathrm{t}}$.

Before LEP startup, the $\mathrm{W}$ and $\mathrm{Z}$ boson masses were measured to be $80.000 \pm$ $0.360 \mathrm{GeV}$ and $91.120 \pm 0.160 \mathrm{GeV}$ respectively. These measurements were equivalent to a precision on the weak mixing angle $\sin ^{2} \theta_{\mathrm{W}}$ of $3.7 \%$ worse than the direct measurement itself: $0.227 \pm 0.006$ corresponding to $2.6 \%$. That is the reason why inputs to the Standard Model fits were $\alpha, G_{\mathrm{F}}$ and $\sin ^{2} \theta_{\mathrm{W}}$. In 1995 , at the end

\footnotetext{
*Presented at the Siena Conference, The Legacy of LEP and SLC, October 8-11 2001, on behalf of the LEP/SLD Collaborations and of the LEP Electroweak Working Group.
}

of LEP-I, the Z boson mass was known with an impressive precision of $2.210^{-5}$ hence replacing $\sin ^{2} \theta_{\mathrm{W}}$ as input parameter to electroweak fits. The Fermi constant $G_{F}$ is determined from the $\mu$ lifetime, $G_{F}=1.16637(1) \cdot 10^{-5} \mathrm{GeV}^{-2}[1]$. The relative error of $G_{F}$ is comparable to that of $m_{\mathrm{Z}}$; both errors have negligible effects on the electroweak fit results.

More details can be found in the 2001 summary of the Electroweak Working Group [2].

\subsection{The top quark mass}

Many of the observables measured at LEP-I and SLC have sensitivity to the top quark mass (typically $20 \mathrm{GeV}$ precision for the measurements of $R_{\mathrm{b}}, \Gamma_{\mathrm{Z}}$ and $\sin ^{2} \theta_{\mathrm{W}}$ ), allowing for an impressive precision on the prediction of the top quark mass as the amount of data collected increased, as shown in Figure 1. In 2001, the top quark mass is measured to be $174.3 \pm 5.1$ $\mathrm{GeV}$ [3] and the electroweak fit yields $181_{-9}^{+11}$ $\mathrm{GeV}$.

\subsection{After $m_{\mathrm{t}}$ ?}

Once the top quark mass is experimentally known, it is used to further constrain the electroweak fits allowing for predictions of $m_{\mathrm{W}}$ and of $m_{\mathrm{H}}$. The first sensitivity curve to the Higgs boson mass (shown as a $\chi^{2}$ curve on Figure 2) was published in the 1994 report of the Electroweak Working Group [4]. 
2

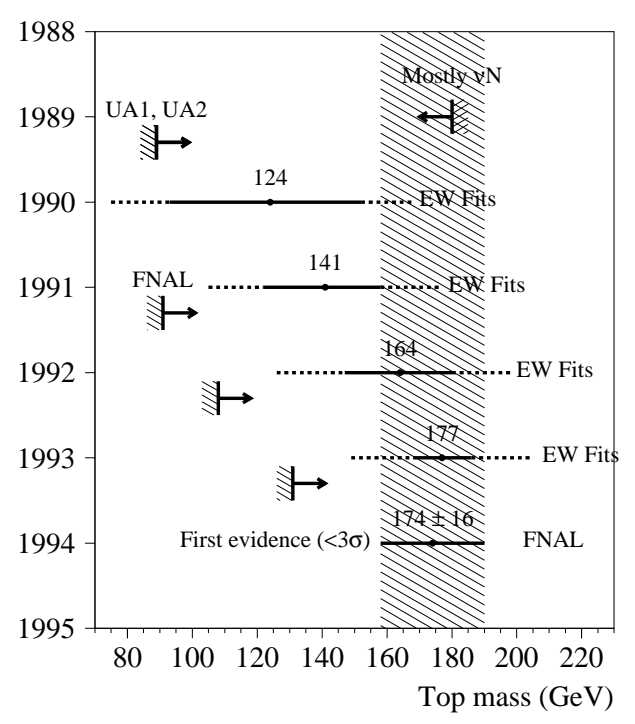

Figure 1. Time evolution of the top quark mass derived from electroweak fits, compared to direct searches results.

\section{A word about a $3.3 \sigma$ effect}

At the time of this note and still for some time, it is difficult to present electroweak fit results without mentioning the so called " $3.3 \sigma$ " effect.

The asymmetry measurements from LEP and SLD are combined into a single parameter, the effective electroweak mixing angle, $\sin ^{2} \theta_{\text {eff }}^{\text {lept }}$, defined as:

$\sin ^{2} \theta_{\mathrm{eff}}^{\mathrm{lept}} \equiv \frac{1}{4}\left(1-\frac{g_{\mathrm{V} \ell}}{g_{\mathrm{A} \ell}}\right)$,

without making strong model-specific assumptions. In Figure 3 are summarised the different measurements. As it can be seen, the combinations based on the leptonic results plus $\mathcal{A}_{\ell}(\mathrm{SLD})$ and on the hadronic forward-backward asymmetries differ by 3.3 standard deviations, mainly caused by the two most precise measurements of $\sin ^{2} \theta_{\mathrm{eff}}^{\mathrm{lept}}, \mathcal{A}_{\ell}(\mathrm{SLD})$ dominated by $A_{\mathrm{LR}}$, and $A_{\mathrm{FB}}^{0, \mathrm{~b}}$ (LEP). The averages of the two group of measurements are indicated in Table 1 . The average of the six measurements leads to $\sin ^{2} \theta_{\text {eff }}^{\text {lept }}=$ $0.23152 \pm 0.00017$ with a $\chi^{2}$ probability of $2.5 \%$. The lepton based average prefers a low $m_{\mathrm{H}}$ value (below $100 \mathrm{GeV}$ ), while the hadronic based aver-

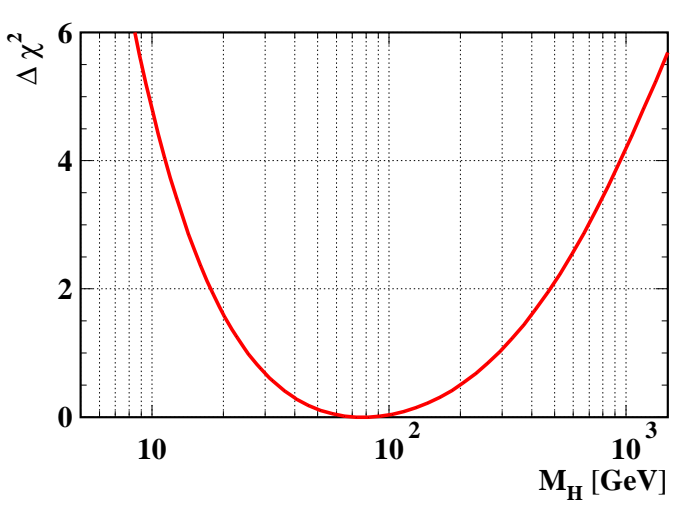

Figure 2. First $\Delta \chi^{2}=\chi^{2}-\chi_{\min }^{2}$ vs $m_{\mathrm{H}}$ fit results plot including the CDF value of $m_{\mathrm{t}}$.

age prefers a high $m_{\mathrm{H}}$. Before any further considerations, one should keep in mind that $3 \sigma$ effects exist in nature. Figure 4 shows the evolution of $\sin ^{2} \theta_{\mathrm{eff}}^{\text {lept }}$ determine at LEP from $A_{\mathrm{FB}}^{0, \mathrm{~b}}$ (right) and SLD from $\mathcal{A}_{\ell}$ (left) as over seven years. As it can be seen, the significance of the discrepancy does not increase with time as the precision of the measurements does, suggesting a statistical fluctuation nature of the difference.

Table 1

Determinations of $\sin ^{2} \theta_{\text {eff }}^{\text {lept }}$ from asymmetries.

\begin{tabular}{|c|c|}
\hline & $\sin ^{2} \theta_{\text {eff }}^{\text {lept }}$ \\
\hline \hline$A_{\mathrm{FB}}^{0, \ell}, \mathcal{A}_{\ell}\left(\mathcal{P}_{\tau}\right), \mathcal{A}_{\ell}(\mathrm{SLD})$ & $0.23113 \pm 0.00021$ \\
\hline$A_{\mathrm{FB}}^{0, \mathrm{~b}}, A_{\mathrm{FB}}^{0, \mathrm{c}},\left\langle Q_{\mathrm{FB}}\right\rangle$ & $0.23230 \pm 0.00029$ \\
\hline
\end{tabular}

\section{Measurements}

On figure 5, the various inputs to the electroweak fits are summarised, also shown the pulls (difference between measurement and fit in units of the total measurement error) of the various measurements. The pulls are derived from the Standard Model fit including all data with the Higgs mass treated as a free parameter. As it can be seen the largest pull $(-2.9)$ if for $A_{\mathrm{FB}}^{0, \ell}$ (reflecting the fact the it is the most precise measurement preferring a high $m_{H}$ value). The pull distribution from all measurements follows a Gaussian law. 


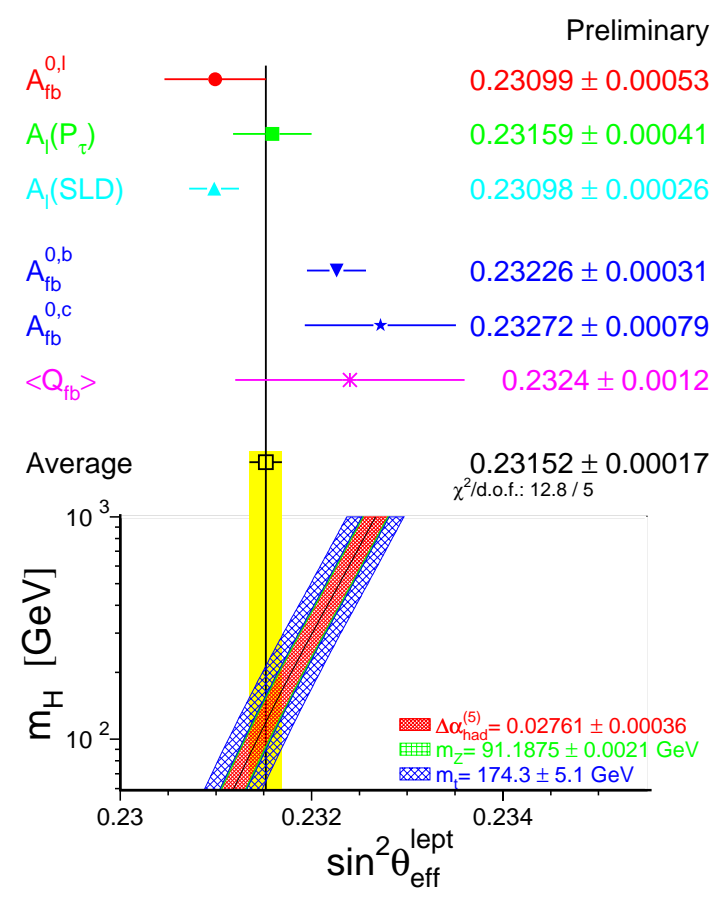

Figure 3. Comparison of several determinations of $\sin ^{2} \theta_{\text {eff }}^{\text {lept }}$ from asymmetries. Also shown is the prediction of the Standard Model as a function of $m_{\mathrm{H}}$. The width of the Standard Model band is due to the uncertainties in $\Delta \alpha_{\text {had }}^{(5)}\left(m_{\mathrm{Z}}^{2}\right), m_{\mathrm{Z}}$ and $m_{\mathrm{t}}$. The total width of the band is the linear sum of these effects.

\section{Theoretical and Parametric Uncertain- ties}

Detailed studies of the theoretical uncertainties in the Standard Model predictions due to missing higher-order electroweak corrections and their interplay with QCD corrections can be found in [5].

The recently calculated complete fermionic two-loop corrections on $m_{\mathrm{W}}$ [6] are currently only used in the determination of the theoretical uncertainty. Their effect on $m_{\mathrm{W}}$ is small compared to the current experimental uncertainty on $m_{\mathrm{W}}$, however, the naive propagation of this new $m_{\mathrm{W}}$ to $\sin ^{2} \theta_{\text {eff }}^{\text {lept }}=\kappa\left(1-m_{\mathrm{W}}^{2} / m_{\mathrm{Z}}^{2}\right)$, keeping the electroweak form-factor $\kappa$ unmodified, shows a more visible effect as $\sin ^{2} \theta_{\text {eff }}^{\text {lept }}$ is measured very precisely. Thus the corresponding calculations for $\sin ^{2} \theta_{\text {eff }}^{\text {lept }}($ or $\kappa)$ and for the partial Z widths are urgently needed; in particular since partial can-

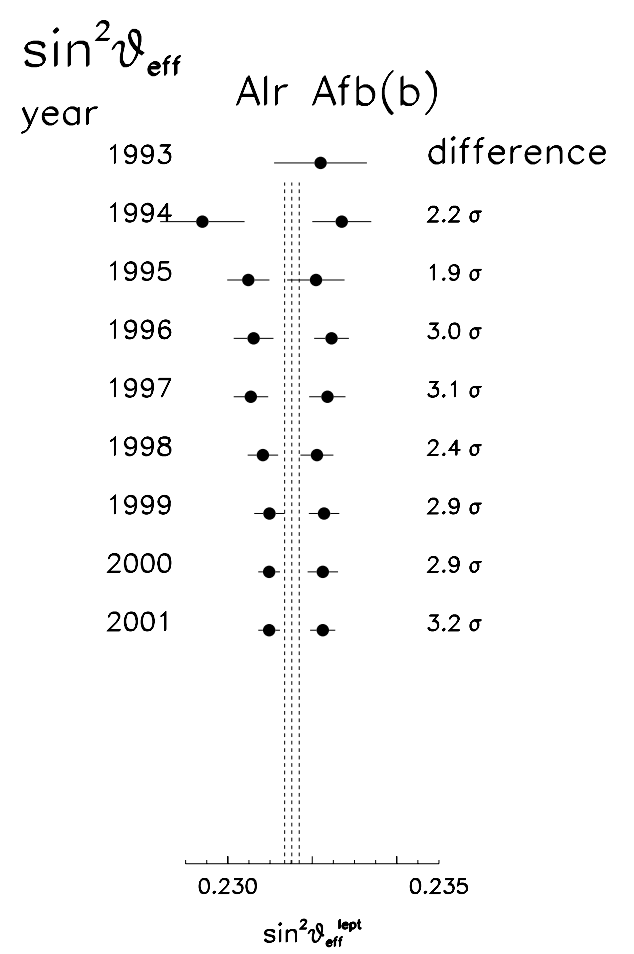

Figure 4. Evolution of $\sin ^{2} \theta_{\text {eff }}^{\text {lept }}$ determine at LEP from $A_{\mathrm{FB}}^{0, \mathrm{~b}}$ (right) and SLD from $\mathcal{A}_{\ell}$ (left) as over seven years.

cellations of these new corrections in the product $\kappa\left(1-m_{\mathrm{W}}^{2} / m_{\mathrm{Z}}^{2}\right)=\sin ^{2} \theta_{\mathrm{eff}}^{\text {lept }}$ are expected [7].

The use of the new QCD corrections[8] increases the value of $\alpha_{\mathrm{S}}\left(m_{\mathrm{Z}}^{2}\right)$ by 0.001 , as expected. The effects of missing higher-order QCD corrections on $\alpha_{\mathrm{S}}\left(m_{\mathrm{Z}}^{2}\right)$ covers missing higher-order electroweak corrections and uncertainties in the interplay of electroweak and QCD corrections and is estimated to be at least 0.002 [9]. The determination of the size of remaining theoretical uncertainties is under continued study.

At present the impact of theoretical uncertainties on the determination of Standard Model parameters from the precise electroweak measurements is small compared to the error due to the uncertainty in the value of $\alpha\left(m_{\mathrm{Z}}^{2}\right)$, which is included in the results.

The uncertainty in $\alpha\left(m_{\mathrm{Z}}^{2}\right)$ arises from the contribution of light quarks to the photon vacuum 


\begin{tabular}{|c|c|c|c|}
\hline \multicolumn{4}{|c|}{ Summer 2001} \\
\hline & Measurement & Pull & 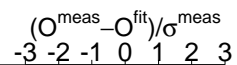 \\
\hline$\overline{\Delta \alpha_{\text {had }}^{(5)}\left(m_{z}\right)}$ & $0.02761 \pm 0.00036$ & -.35 & - \\
\hline $\mathrm{m}_{\mathrm{z}}[\mathrm{GeV}]$ & $91.1875 \pm 0.0021$ & .03 & \\
\hline$\Gamma_{\mathrm{Z}}[\mathrm{GeV}]$ & $2.4952 \pm 0.0023$ & -.48 & 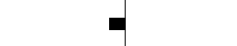 \\
\hline$\sigma_{\text {had }}^{0}[n b]$ & $41.540 \pm 0.037$ & 1.60 & \\
\hline $\mathrm{R}_{1}$ & $20.767 \pm 0.025$ & 1.11 & \\
\hline$A_{\mathrm{fb}}^{0, I}$ & $0.01714 \pm 0.00095$ & .69 & \\
\hline$A_{1}\left(P_{\tau}\right)$ & $0.1465 \pm 0.0033$ & -.54 & 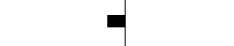 \\
\hline $\mathrm{R}_{\mathrm{b}}$ & $0.21646 \pm 0.00065$ & 1.12 & \\
\hline $\mathrm{R}_{\mathrm{c}}$ & $0.1719 \pm 0.0031$ & -.12 & I \\
\hline$A_{f b}^{0, b}$ & $0.0990 \pm 0.0017$ & -2.90 & \\
\hline$A_{f b}^{0, c}$ & $0.0685 \pm 0.0034$ & -1.71 & \\
\hline$A_{b}$ & $0.922 \pm 0.020$ & -.64 & - \\
\hline$A_{c}$ & $0.670 \pm 0.026$ & .06 & \\
\hline$A_{1}(S L D)$ & $0.1513 \pm 0.0021$ & 1.47 & $\boldsymbol{\sigma}$ \\
\hline $\sin ^{2} \theta_{\text {eff }}^{\text {lept }}\left(Q_{\mathrm{fb}}\right)$ & $0.2324 \pm 0.0012$ & .86 & \\
\hline $\mathrm{m}_{\mathrm{W}}^{(\mathrm{LEP})}[\mathrm{GeV}]$ & $80.450 \pm 0.039$ & 1.32 & \\
\hline$m_{t}[\mathrm{GeV}]$ & $174.3 \pm 5.1$ & -.30 & a \\
\hline $\mathrm{m}_{\mathrm{W}}^{(\mathrm{TEV})}[\mathrm{GeV}]$ & $80.454 \pm 0.060$ & .93 & \\
\hline $\sin ^{2} \theta_{w}(v N)$ & $0.2255 \pm 0.0021$ & 1.22 & \\
\hline$Q_{w}(C s)$ & $-72.50 \pm 0.70$ & .56 & 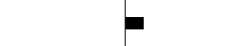 \\
\hline
\end{tabular}

Figure 5. Summary of measurements that enter the electroweak fits together with their pull.

polarisation $\left(\Delta \alpha_{\text {had }}^{(5)}\left(m_{\mathrm{Z}}^{2}\right)\right)$ :

$\alpha\left(m_{\mathrm{Z}}^{2}\right)=\frac{\alpha(0)}{1-\Delta \alpha_{\ell}\left(m_{\mathrm{Z}}^{2}\right)-\Delta \alpha_{\text {had }}^{(5)}\left(m_{\mathrm{Z}}^{2}\right)-\Delta \alpha_{\mathrm{top}}\left(m_{\mathrm{Z}}^{2}\right)}$,

where $\alpha(0)=1 / 137.036$. The top contribution, $-0.00007(1)$, depends on the mass of the top quark, and is therefore determined inside the electroweak libraries. The leptonic contribution is calculated to third order[10] to be 0.03150 , with negligible uncertainty.

The new evaluation of the hadronic contribution $0.02761 \pm 0.0036$ which takes into account the recently published results on electronpositron annihilations into hadrons at low centreof-mass energies by the BES collaboration [11] is used. The uncertainty translates into an error of 0.00013 on the Standard Model prediction of $\sin ^{2} \theta_{\text {eff }}^{\text {lept }}$, and errors of $0.2 \mathrm{GeV}$ and 0.1 on the fitted values of $m_{\mathrm{t}}$ and $\log \left(m_{\mathrm{H}}\right)$, all included in the results presented.
There are also several evaluations of $\Delta \alpha_{\text {had }}^{(5)}\left(m_{\mathrm{Z}}^{2}\right)[12-19]$ which are more theory-driven. One of the most recent ones (Reference [18]) also includes the new results from BES, yielding $0.02738 \pm 0.00020$. To show the effects of the uncertainty of $\alpha\left(m_{\mathrm{Z}}^{2}\right)$, this evaluation of the hadronic vacuum polarisation is also used.

\section{Fit results}

Most observables have sensitivity to the Higgs boson mass as it can be seen in Figure 6. The most sensitive measurements are the asymmetries, i.e., $\sin ^{2} \theta_{\text {eff }}^{\text {lept }}$ and the $\mathrm{W}$ mass. A reduced uncertainty for the value of $\alpha\left(m_{\mathrm{Z}}^{2}\right)$ would therefore result in an improved constraint on $\log m_{\mathrm{H}}$ and thus $m_{\mathrm{H}}$.

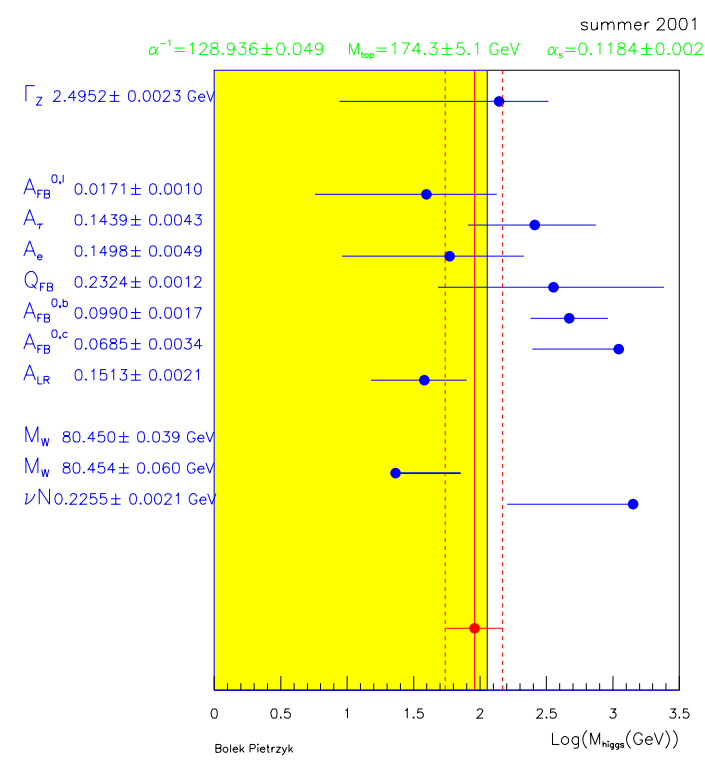

Figure 6. Constraints on $\log m_{\mathrm{H}}$ from various observables.

Several different Standard Model fits to the data are discussed. The predictions are calculated with TOPAZ0 [20] and ZFITTER [21].

The data can first be used to determine the top quark and $\mathrm{W}$ masses indirectly, which can be compared to the direct measurements performed at the $\mathrm{p} \overline{\mathrm{p}}$ colliders and LEP-II, all the results ex- 
cept the LEP-II and $\mathrm{p} \overline{\mathrm{p}}$ colliders $m_{\mathrm{W}}$ and $m_{\mathrm{t}}$ results are used. The indirect measurements of $m_{\mathrm{W}}$ and $m_{\mathrm{t}}$ are shown in Figure 7, compared with the direct measurements. Also shown are the Standard Model predictions for Higgs masses between 114 and $1000 \mathrm{GeV}$. As can be seen in the figure, the indirect and direct measurements of $m_{\mathrm{W}}$ and $m_{\mathrm{t}}$ are in good agreement, and both sets prefer a low value of the Higgs mass

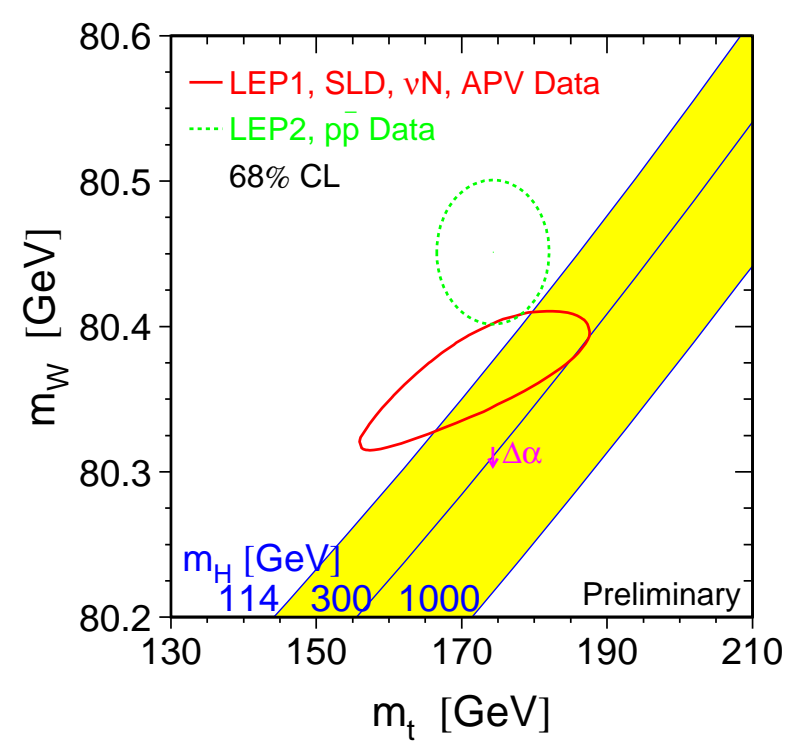

Figure 7. The comparison of the indirect measurements of $m_{\mathrm{W}}$ and $m_{\mathrm{t}}$ (solid contour) and the direct measurements (dashed contour). In both cases the $68 \%$ CL contours are plotted. Also shown is the Standard Model relationship for the masses as a function of the Higgs mass.

From a fit to all data except $m_{\mathrm{W}}$, the best indirect determination of $m_{\mathrm{W}}$ is obtained. The indirect determination of $\mathrm{W}$ boson mass $80.373 \pm$ $0.023 \mathrm{GeV}$ is in agreement with the combination of direct measurements from LEP-II and $\mathrm{p} \overline{\mathrm{p}}$ colliders [22] of $m_{\mathrm{W}}=80.451 \pm 0.033 \mathrm{GeV}$.

Similarly, the indirect determination of the top quark mass : $m_{\mathrm{t}}=181_{-9}^{+11} \mathrm{GeV}$, is in very good agreement with the direct measurement of $m_{\mathrm{t}}=$ $174.3 \pm 5.1 \mathrm{GeV}$.
Finally, the best constraints on $m_{\mathrm{H}}$ are obtained when all data are used in the fit. In Figure 8 the observed value of $\Delta \chi^{2} \equiv \chi^{2}-\chi_{\min }^{2}$ as a function of $m_{\mathrm{H}}$ is plotted for the fit including all data. The $\chi^{2}$ per degree of freedom of the fit is $23 / 15$ corresponding to a probability of $8 \%$. The solid curve is the result using ZFITTER. The result is $\log \left(m_{\mathrm{H}} / \mathrm{GeV}\right)=1.94 \pm 0.21$, corresponding to $m_{\mathrm{H}}=88_{-35}^{+53} \mathrm{GeV}$. The shaded band represents the uncertainty due to uncalculated higher-order corrections, as estimated by ZFITTER and TOPAZ0. Compared to previous analyses, its width is enlarged towards lower Higgs-boson masses due to the effects of the complete fermionic two-loop calculation of $m_{\mathrm{W}}$ discussed above. The 95\% confidence level upper

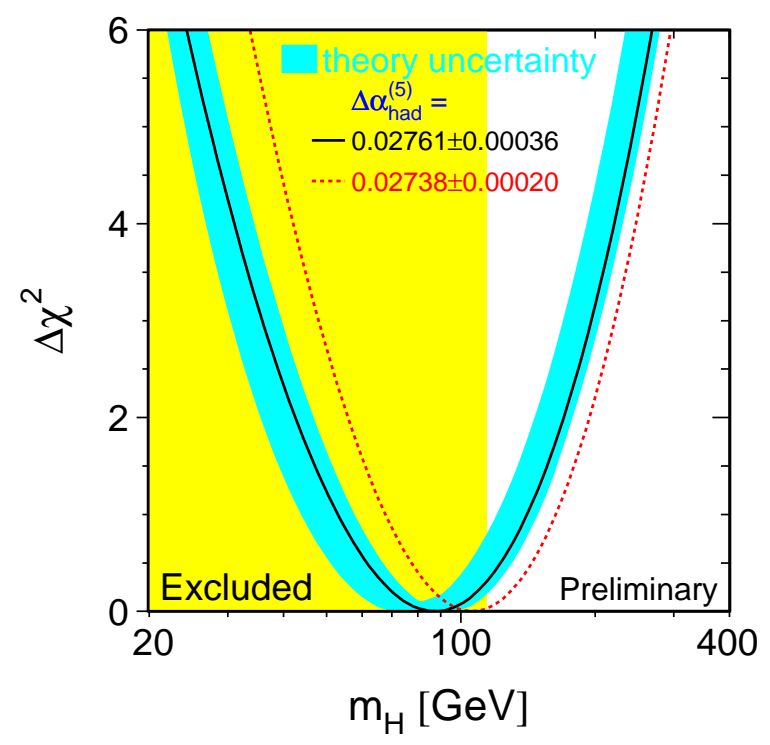

Figure 8. $\Delta \chi^{2}=\chi^{2}-\chi_{\min }^{2}$ vs. $m_{\mathrm{H}}$ curve. The line is the result of the fit using all data; the band represents an estimate of the theoretical error due to missing higher order corrections. The vertical band shows the $95 \% \mathrm{CL}$ exclusion limit on $m_{\mathrm{H}}$ from the direct search.

limit on $m_{\mathrm{H}}$ (taking the band into account) is 196 $\mathrm{GeV}$. The lower limit on $m_{\mathrm{H}}$ of approximately 114 $\mathrm{GeV}$ obtained from direct searches[23] is not used 
in the determination of this limit. Also shown is the result (dashed curve) obtained when using $\Delta \alpha_{\text {had }}^{(5)}\left(m_{\mathrm{Z}}^{2}\right)$ of Reference [18]. That fit results in $\log \left(m_{\mathrm{H}} / \mathrm{GeV}\right)=2.03 \pm 0.19$ corresponding to $m_{\mathrm{H}}=106_{-38}^{+57} \mathrm{GeV}$ and an upper limit on $m_{\mathrm{H}}$ of approximately $222 \mathrm{GeV}$ at $95 \%$ confidence level.

The variation of the upper limit on $m_{\mathrm{H}}$ against experimental parametric errors is tested in Figure 9 , where $\Delta \chi^{2}$ curves corresponding to one sigma variation of $\Delta \alpha_{\text {had }}^{(5)}\left(m_{\mathrm{Z}}^{2}\right)$ and $m_{\mathrm{t}}$ are plotted. Although the position of the minimum changes because of the relative changes of the different measurement in the fit, the upper limit on $m_{\mathrm{H}}$ does not increase above $300 \mathrm{GeV}$.

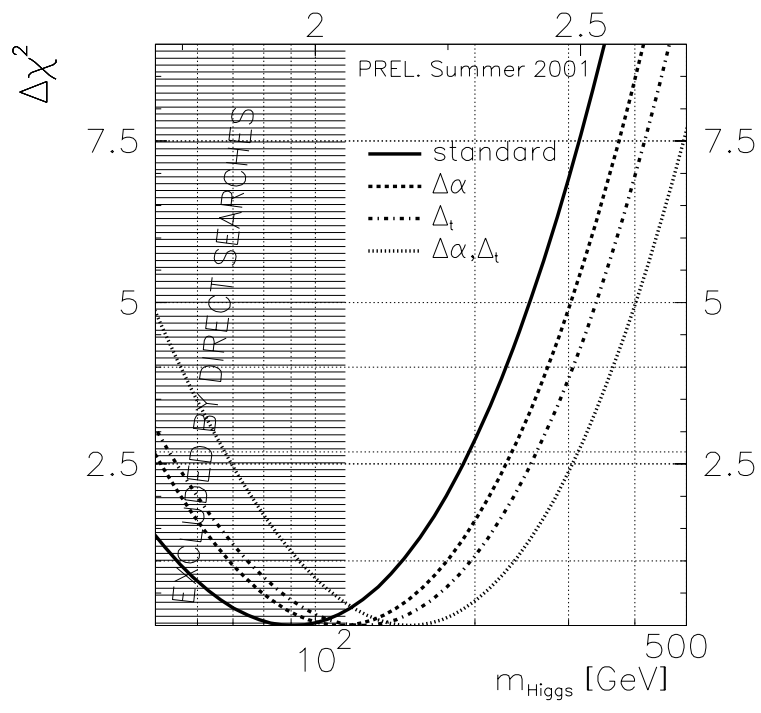

Figure 9. $\Delta \chi^{2}$ for one sigma change of $\Delta \alpha_{\text {had }}^{(5)}\left(m_{\mathrm{Z}}^{2}\right)$ or $m_{\mathrm{t}}$ and both.

\section{Conclusions and Perspectives}

The measurement of $m_{\mathrm{W}}$ at LEP-II is likely to reach a precision of $\approx 25 \mathrm{MeV}$, not too far from the uncertainty on the prediction obtained via the radiative corrections of the $\mathrm{Z}$ data, providing a further important test of the Standard Model. As the LEP energy final value is not yet known, the absolute $m_{\mathrm{W}}$ mass value from LEP might change. However to further, significantly, con- strain $m_{\mathrm{H}}$ a more accurate measurement of $m_{\mathrm{t}}$ is mandatory. A precision of $2 \mathrm{GeV}$ is likely to be obtained at FERMILAB run II. Figure 10 shows the $\Delta \chi^{2}$ curves that would be obtained with such precisions, without any change in central values of measurements. A precision of about $20 \mathrm{GeV}$ on $m_{\mathrm{H}}$ is reachable.

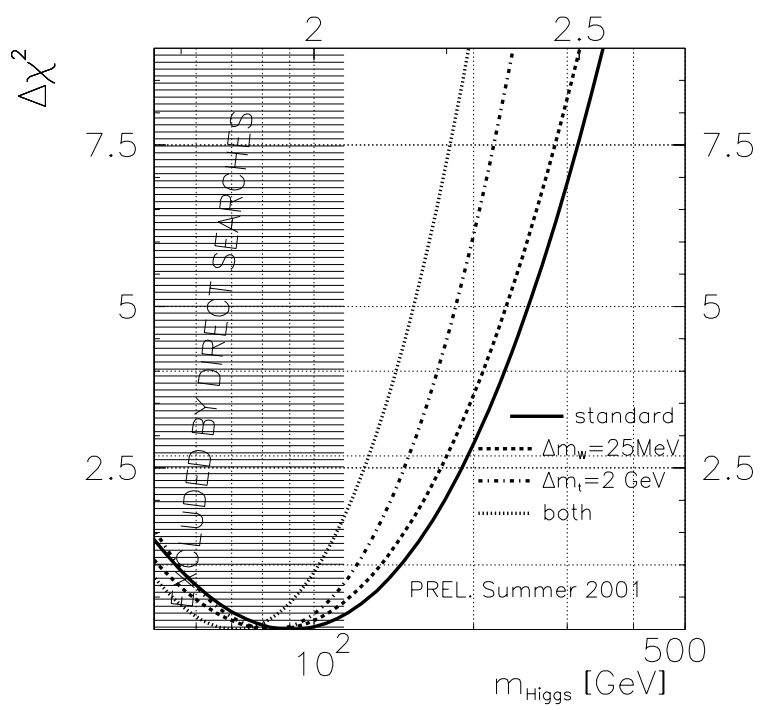

Figure 10. $\Delta \chi^{2}$ curves for a precision of 25 $\mathrm{MeV}$ on $m_{\mathrm{W}}$ and $2 \mathrm{GeV}$ on $m_{\mathrm{t}}$. Central values of $m_{\mathrm{W}}$ and $m_{\mathrm{t}}$ are kept unchanged.

Figure 11 shows the evolution of both the lower limit on the Higgs boson mass from direct searches (hatched area) and the upper limit from electroweak fits (dashed area) as a function of time. The gap is not closed, a slightly longer run for LEP with a bit higher energy might have closed it.

Electroweak fits from LEP and SLC data have allowed to test the internal consistency of the Standard Model with great precision, three to five times better than anticipated. The top quark mass was predicted several years before it has been discovered. The measurements led to the prediction of a relatively light Higgs boson (around $100 \mathrm{GeV})$, with the same precision $(\approx$ $50 \%)$ as on the top quark mass before LEP and SLC startup. 


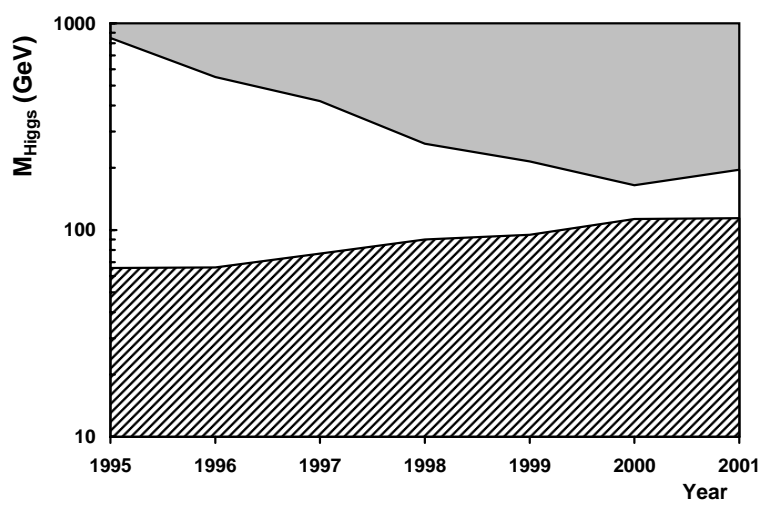

Figure 11. Variation over seven years of the lower limit on the Higgs boson mass from direct searches (dashed area) compared to the upper limit derived from electroweak fits (shaded area).

\section{REFERENCES}

1. T. van Ritbergen and R.G. Stuart, Phys. Rev. Lett. 82 (1999) 488.

2. The LEP Collaborations ALEPH, DELPHI, L3 and OPAL, the LEP Electroweak Working Group, the SLD Heavy Flavour Group and Electroweak Working Group, A Combination of Preliminary Electroweak Measurements and Constrains on the Standard Model , hep-ex/0112021.

3. The Top Averaging Group, L. Demortier et al., for the $\mathrm{CDF}$ and $\mathrm{D} \varnothing$ Collaborations, FERMILAB-TM-2084 (1999).

4. The LEP Collaborations ALEPH, DELPHI, L3, OPAL and the LEP Electroweak Working Group, Combined Preliminary Data on $\mathrm{Z}$ Parameters from the LEP Experiments and Constraints on the Standard Model, CERNPPE/94-187.

5. D. Bardin, M. Grünewald and G. Passarino, Precision Calculation Project Report, hep$\mathrm{ph} / 9902452$.

6. A. Freitas, W. Hollik, W. Walter and G. Weiglein, Phys. Lett. B495 (2000) 338.

7. D. Bardin, P. Gambino, G. Passarino, G. Weiglein, private communication, spring 2001.

8. A. Czarnecki and J. Kühn, Phys. Rev. Lett. 77 (1996) 3955;
R. Harlander, T. Seidensticker and M. Steinhauser, Phys. Lett. B426 (1998) 125.

9. T. Hebbeker, M. Martinez, G. Passarino and G. Quast, Phys. Lett. B331 (1994) 165;

P.A. Raczka and A. Szymacha, Phys. Rev. D54 (1996) 3073;

D.E. Soper and L.R. Surguladze, Phys. Rev. D54 (1996) 4566.

10. M. Steinhauser, Phys. Lett. B429 (1998) 158.

11. The BES Collaboration, J.Z.Bai et al., Measurements of the Cross Section for $e^{+} e^{-} \rightarrow$ hadrons at Center-of-Mass Energies from 2 to $5 \mathrm{GeV}$, hep-ex/0102003.

12. M. L. Swartz, Phys. Rev. D53 (1996) 5268.

13. A.D. Martin and D. Zeppenfeld, Phys. Lett. B345 (1994) 558.

14. R. Alemany, et al., Eur. Phys. J. C2 (1998) 123.

15. M. Davier and A. Höcker, Phys. Lett. B419 (1998) 419.

16. J.H. Kühn and M. Steinhauser, Phys. Lett. B437 (1998) 425.

17. J. Erler, Phys. Rev. D59, (1999) 054008.

18. A. D. Martin, J. Outhwaite and M. G. Ryskin, Phys. Lett. B492 (2000) 69.

19. F. Jegerlehner, Hadronic Effects in (g-2) and $\alpha_{Q E D}\left(M_{Z}\right)$ : Status and Perspectives, Proc. of Int. Symp. on Radiative Corrections, Barcelona, Sept. 1998, page 75.

20. G. Montagna et al., Comput. Phys. Commun. 117 (1999) 278;

http://www.to.infn.it/ giampier/topaz0.html .

21. D. Bardin et al., Z. Phys. C44 (1989) 493; Comp. Phys. Comm. 59 (1990) 303; Nucl. Phys. B351(1991) 1; Phys. Lett. B255 (1991) 290 and CERN-TH 6443/92 (May 1992); the most recent version of ZFITTER (6.21) is described in DESY 99-070, hep-ph/9908433 (Aug 1999) published in Comp. Phys. Comm. 133 (2001) 229.

22. http://www-cdf.fnal.gov/physics/ewk/wmass_new.htm.

23. P. Igo-Kemenes, these proceedings. 